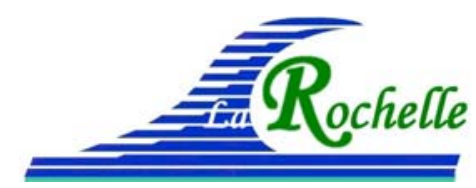

XVèmes Journées Nationales Génie Côtier - Génie Civil

La Rochelle, 29 au 31 mai 2018

DOI:10.5150/jngcgc.2018.044 (C) Editions Paralia CFL

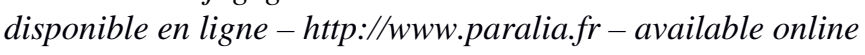

\title{
Mesures in situ des impacts de vagues sur une digue composite : résultats préliminaires
}

\author{
Pierre-Antoine PONCET ${ }^{1}$, Stéphane ABADIE ${ }^{1}$, Benoit LARROQUE ${ }^{1}$, \\ Damien SOUS ${ }^{3}$, Benoit LIQUET ${ }^{2}$
}

1. UNIV PAU \& PAYS ADOUR/ E2S UPPA, Laboratoire des Sciences de l'Ingénieur Appliquées à la Mécanique et au génie Électrique - IPRA, EA4581, 64600, Anglet, France.pa.poncet@univ-pau.fr

2. CNRS/UNIV PAU \& PAYS ADOUR/E2S UPPA, Laboratoire de Mathématiques et de leurs Applications de Pau - Fédération*, UMR5142 64000, Pau, France.

3. MIO-USTV/AMU/CNRS/IRD, UM 110, Université du sud-Toulon-Var, La Garde, France.

\section{Résumé :}

Afin d'étudier les pressions d'impact sur la digue de l'Artha à Saint-Jean de Luz, l'ouvrage a été équipé de deux capteurs de pression. Les deux capteurs ont enregistré le signal de pression à $10 \mathrm{kHz}$ par tranches de 10 minutes toutes les heures. Par ailleurs le champ de vagues, le niveau d'eau et le vent sont connus. Le jeu de données couvre les mois de Janvier à Avril 2016. Les mesures de pression sont en accord avec des études similaires précédentes (DE ROUVILLE, 1938), (BULLOCK, 2003), mettant en évidence des pics de pression significativement plus faibles que ceux obtenus en canal (HOFLAND, 2011) ou par simulation numérique. Un des objectifs de cette étude est d'identifier les conditions de houle, de niveau d'eau et de vent qui génèrent les impacts les plus destructeurs. L'influence principale de la hauteur de vague sur la variabilité en pressions est mise en évidence pour ce jeu de données. Le niveau d'eau et l'orientation de la houle étant les paramètres suivants les plus influents. Une première analyse impact par impact est proposée.

Mots-clés : Vague, Impact, Digue, Mesures in situ.

\section{Introduction}

Les impacts de vagues sur les structures côtières sont la source de nombreux dégâts et ont donc été étudiés à de nombreuses reprises. Plusieurs mécanismes entrent en jeux lors de ce phénomène complexe. Les pressions générées lors de l'impact sont majoritairement gouvernées par la forme locale de la surface libre. Ainsi des pressions très élevées peuvent être générées lorsque le front d'onde est quasiment parallèle à la surface impactée, (WAGNER, 1932). Ce type d'impact, qui sont les plus violents en terme de pression sur l'ouvrage, génèrent un jet vertical très rapide associés à de fort gradients de pression (COOKER \& PEREGRINE, 1990 \& 1992). Les vagues déferlantes peuvent aussi piéger un volume d'air plus ou moins important lorsqu'elles 


\section{Thème 3 - Instrumentation, mesures, imagerie et télédétection}

interagissent avec la paroi verticale. Cette poche de gaz interagi avec la surface libre ce qui module les pressions d'impact. Mais l'air peut aussi être entraîné sous forme de petites bulles. La compressibilité globale de l'eau est alors augmentée conduisant à des effets complexes capables d'amplifier les pics de pression. De nombreuses études expérimentales ont été menées pour explorer ce processus complexe (BULLOCK et al., 2007 ; HOFLAND et al., 2011 ; KAMINSKI \& BOGAERT, 2009).

Mais les travaux expérimentaux s'écartent du processus naturel. Premièrement, le processus naturel est essentiellement 3D alors que les expériences en canal sont bidimensionnelles. Cela pourrait entraîner une surestimation ou une sous-estimation de la pression maximale (PEREGRINE, 2003). En second lieu, la règle d'échelle appropriée est difficile à établir (BULLOCK et al., 2001) bien que les expériences récentes se rapprochent de l'échelle réelle (HOFLAND et al., 2011). Troisièmement, les empilements de blocs dissipent une grande partie de l'énergie incidente, (BOUGIS et al., 2016) ont réalisé des essais au 1/30 ème et au 1/60 ème d'impact de vagues sur la digue de l'Artha et de Socoa prenant en compte ces enrochements et la topographie de l'ouvrage. Enfin certaines variables environnementales (orientation de la houle, période, niveau d'eau, vent) ne sont pas systématiquement prises en compte dans les études en laboratoire. Pour toutes ces raisons, il est nécessaire de prendre des mesures terrain de la pression d'impact des vagues.

Parmi les premières mesures terrain, DE ROUVILLE et al., (1938) ont enregistrées des pressions variant de 1,8 à 6,9 bars pour des hauteurs de vague entre $1.50 \mathrm{~m}$ et $4.50 \mathrm{~m}$. Plus récemment, (BIRD et al. 1998) ont mis au point un capteur spécifique pour enregistrer les impacts des vagues sur les parois verticales, mesurant à la fois la pression et la concentration en air. Ils ont effectué des mesures à la digue d'Alderney avec des valeurs jusqu' à 3,96 bars (BULLOCK et al., 2001) avec un Hmax de 2.88 m. Cette étude présente les résultats préliminaires de quatre mois de mesures en deux points de la digue de l'Artha.

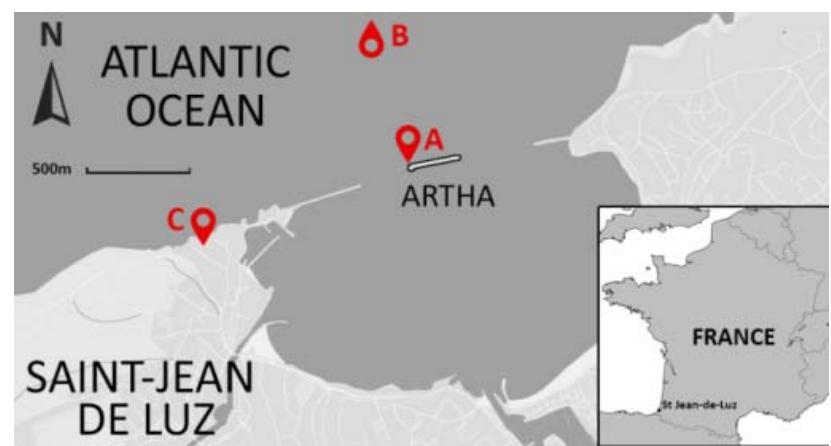

Figure 1. Situation du site d'étude dans la baie de St Jean de Luz avec la position des capteurs. (A : digue et capteurs de pression, B : bouée de houle, $C$ : station météo). 


\section{XVèmes Journées Nationales Génie Côtier - Génie Civil \\ La Rochelle, 29 au 31 mai 2018}

\section{Méthodes}

La structure étudiée dans ce travail est la digue de l'Artha situé à Saint Jean de Luz (figures 1, 2 et 3). La digue est composée d'un caisson entouré d'une berme en béton. Ces deux éléments reposent sur des blocs de béton parallélépipédique de 50 tonnes.

Le dispositif expérimental a été déployé en novembre 2015. Tout d'abord, deux capteurs de pression ont été intégrés dans le mur faisant face au large, à l'endroit où les vagues frappent avec le plus d'énergie (figure 2).

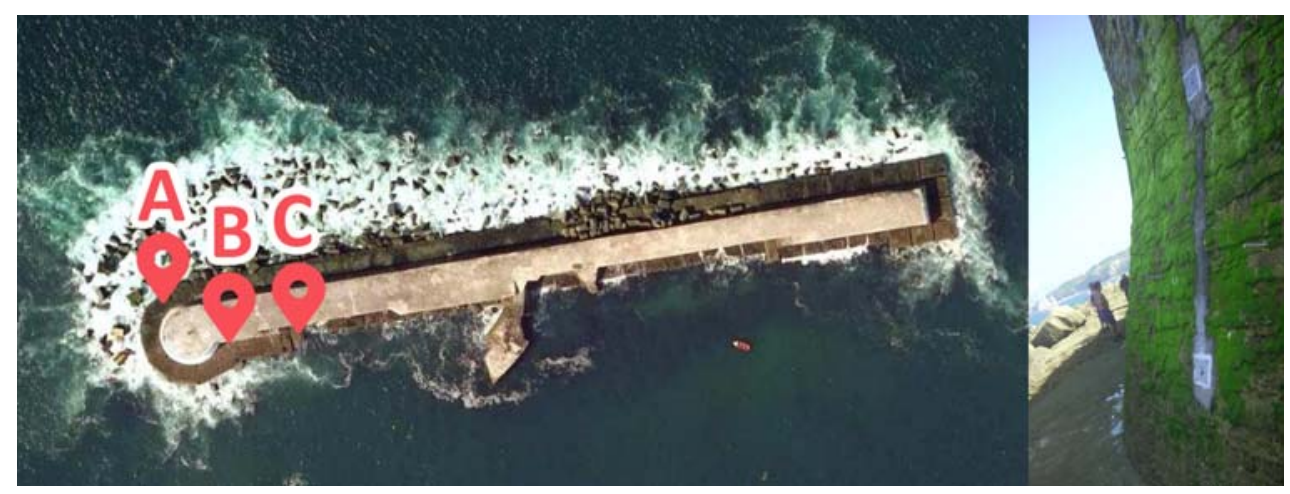

Figure 2. à gauche : Vue de la digue de l'Artha et emplacement du dispositif de mesure (A : capteurs de pression (sur le musoir ouest), B : système d'acquisition, $C$ : panneau photovoltaïque). À droite, photo des deux capteurs de pression affleurant sur la structure.

Le système d'acquisition et de transmission des données se compose de trois parties principales : l'alimentation électrique, le système d'acquisition et la transmission des données (LARROQUE et al., 2018). Par défaut, le système enregistre automatiquement les mesures des capteurs au début de chaque heure pendant 10 minutes à $10 \mathrm{kHz}$ aux deux capteurs du haut et du bas (figure 3).

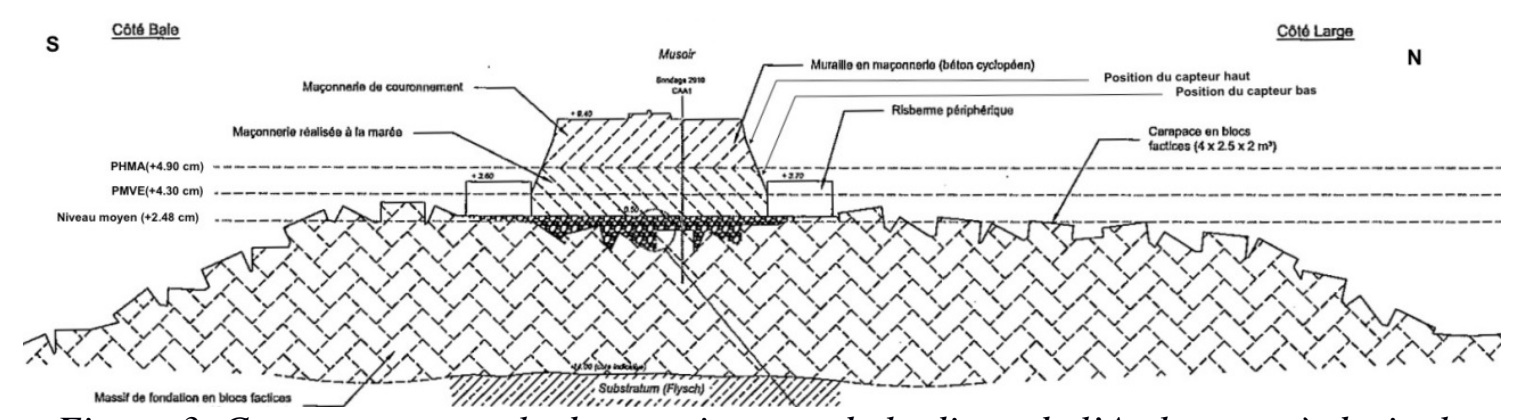

Figure 3. Coupe transversale du musoir ouest de la digue de l'Artha avec à droite la partie exposée au large et à gauche la partie orienté coté baie de St Jean de Luz

Le signal de houle est mesurée grâce à une bouée de surface directionnelle Datawell appartenant au réseau Candhis située à environ $1000 \mathrm{~m}$ à l'ouest (figure 1), par $20 \mathrm{~m}$ de 


\section{Thème 3 - Instrumentation, mesures, imagerie et télédétection}

profondeur. Cette bouée fournit le signal brut (i.e. l'élévation instantanée de la surface libre) ainsi que les paramètres statistiques et spectraux. La vitesse et la direction du vent (à $10 \mathrm{~m}$ d'altitude) ont été mesurées à partir de la station Météo France du sémaphore de Socoa en prenant le maximum sur $10 \mathrm{~min}$, toutes les heures. Enfin, le niveau d'eau a été donné par un modèle mathématique (ARNOUX et al., 2018) basé sur une analyse harmonique des données recueillies localement au marégraphe de Socoa et un terme de correction pour tenir compte de l'effet de pression atmosphérique.

\section{Résultats}

Une étude statistique des conditions environnementales pour chaque échantillon de 10 minutes a été menée. Il en ressort que $58 \%$ de la variabilité en pression est expliquée par les variables environnementales. La variable la plus importante est la hauteur de vague qui explique $45 \%$ de la variabilité totale. Le niveau d'eau explique $10 \%$, la direction de la houle $2,5 \%$ et la période des vagues moins de $0,5 \%$ de la variabilité totale. L'influence du vent (vitesse et direction) ne semble pas significative (LARROQUE et al., 2018).

Tableau 1. Synthèse des paramètres environnementaux et des mesures.

\begin{tabular}{lll}
\hline Paramètre & Valeur moyenne & Valeur maximale \\
\hline$H_{1 / 3}$ & $1.8 \mathrm{~m}$ & $7.57 \mathrm{~m}$ \\
\hline TH $1 / 3$ & 10 secondes & $18 \mathrm{secondes}$ \\
\hline $\begin{array}{l}\text { Theta (orientation de la } \\
\text { houle) }\end{array}$ & \\
\hline Niveau d'eau & $1.7 \mathrm{~m} / 3.5 \mathrm{~m}$ & \\
\hline Vitesse du vent & $8 \mathrm{~m} . \mathrm{s}^{-1}$ & $4.9 \mathrm{~m}$ \\
\hline Orientation du vent & 239 degrés & $26{\mathrm{~m} . \mathrm{s}^{-1}}^{-13}$ \\
\hline$P_{\max }$ (capteur bas) & 0.64 bar & $2.59 \mathrm{bar}$ \\
\hline$P_{\max }$ (capteur haut) & $0.32 \mathrm{bar}$ & $2.69 \mathrm{bar}$ \\
\hline
\end{tabular}

Afin de mieux étudier les différents types d'impacts les échantillons de 10min ont été découpés en impacts unitaires. Seuls les impacts dépassant le seuil de 1 bar (en relatif) ont été retenus. Ce filtre a permis d'obtenir une base de données de 145 impacts. 6 ont été éliminés car ils correspondaient à des artefacts (une seule valeur, supérieure à 30 bars et pas d'impact correspondant détecté sur l'autre capteur). Les signaux pour lesquels l'identification des maxima et minima était ambiguë à cause du bruit ou d'une typologie trop particulière ont été mis à part. Cela permet de retenir une base de données de 81 impacts de plus de 1 bar en relatif avec une identification non ambiguë des extrema. 


\section{XVèmes Journées Nationales Génie Côtier - Génie Civil La Rochelle, 29 au 31 mai 2018}

Parmi les impacts mis à part, 8 présentent une typologie similaire aux impacts à forte aération décrit dans (BULLOCK et al., 2007) et présentés en figure 3. Une analyse spectrale sur les intervalles de temps où les oscillations sont observés, révèle des fréquences pic entre 5 et $50 \mathrm{~Hz}$. Les pressions générées par ce type d'impact semblent couvrir une plage de valeurs assez large allant des pressions moyennes aux plus fortes du jeu de donné. Pour les 81 autres impacts qui présentent des pics plus larges (on les qualifiera d'impact classique par la suite) les oscillations sont moins marquées et moins localisées il est donc plus délicat de réaliser la même analyse spectrale.

Pour essayer de différencier rigoureusement des classes d'impact, deux indices ont été étudiés. Dans un premier temps le rapport palier quasi-hydrostatique / pic percussif. Les 8 impacts atypiques présentent des rapports avec une moyenne à 0.1 et une déviation standard de 3,5\% alors que les impacts classiques présentent un rapport moyen de 0.6 mais une déviation standard de $26 \%$. La question est donc de savoir s'il s'agit réellement de phénomènes différents comme décrits par (OUMERACI et al. 1993 ; HULL \& MÜLLER 2002 ; BULLOCK et al. 2007), ou s'il y a dans la nature un continuum de processus. Un deuxième indice a ensuite été étudié : le rapport entre la pression du pic percussif et le $\mathrm{H}_{1 / 3}$ pendant l'événement (ramenée à une pression hydrostatique). Cet indice met en évidence cinq impacts qui se détachent particulièrement du reste de la distribution, ces cinq impacts étant des impacts qui présentent la forme "forte aération". Il reste donc trois autres impacts qui se distinguaient par le premier indice mais qui ne passent pas le critère de ce nouvel indice. En figure 4 on retrouve les 5 impacts qui présentent des pressions élevées pour des $\mathrm{H}_{1 / 3}$ faibles et pour des niveaux d'eau supérieurs à $4 \mathrm{~m}$.
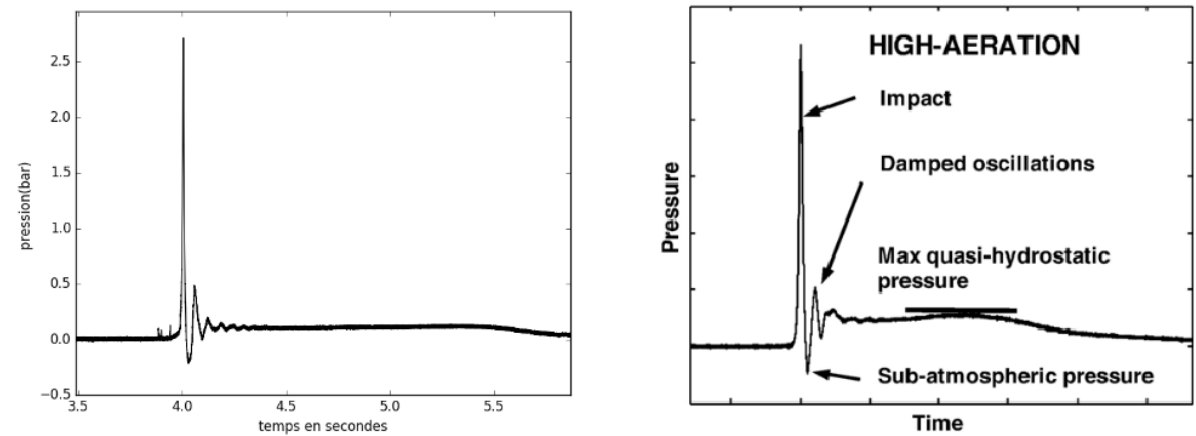

Figure 3. À̀ gauche signal de pression enregistré sur la digue de l'Artha le 04/07/2016 à 15h02. À droite : "Représentation schématique d'un impact haute aération" (BULLOCK et al., 2007).

\section{Discussion}

Dans cet article, de nouvelles mesures in-situ de la pression d'impact des vagues sur un caisson soumis à un climat de vagues énergétiques ont été présentées. Les valeurs de 


\section{Thème 3 - Instrumentation, mesures, imagerie et télédétection}

pression maximale enregistrées au cours de l'expérience sur le terrain sont un ordre de grandeur inférieur aux pressions enregistrées en canal. Plusieurs raisons peuvent expliquer ce résultat. Les blocs forcent la plupart des vagues à déferler suffisamment avant le mur du caisson et à dissiper leur énergie (ces blocs ont une structure 3D qui influence le déferlement et qui n'as pu être prise en compte dans les expériences de (BOUGIS et al., 2016). Deuxièmement, la digue de l'Artha est orientée selon un axe est-ouest alors que la plupart des vagues viennent de l'ouest-nord-ouest. Pour capter les pressions maximales, des capteurs ont été placés sur la partie ouest du brise-lames, sur une partie courbée du mur. Pour cette raison, les pressions générées peuvent être réduites par rapport à une paroi plane, les crêtes n'arrivant pas parallèlement à la digue. Un modèle linéaire multivarié a été mis en œuvre pour étudier les liens entre la pression d'impact maximal et les paramètres environnementaux. La hauteur de houle significative explique à elle seule $45 \%$ de la variabilité avec ce modèle. Les résultats présentés en figure 4 vont être approfondis en utilisant d'autres indices et variables. En particulier le Hs en pied d'ouvrage déterminé par une formule de shoalling permettrait de prendre en compte la période également. Les travaux de modélisation en cours devraient également permettre cette étude ainsi que l'analyse vague à vague.

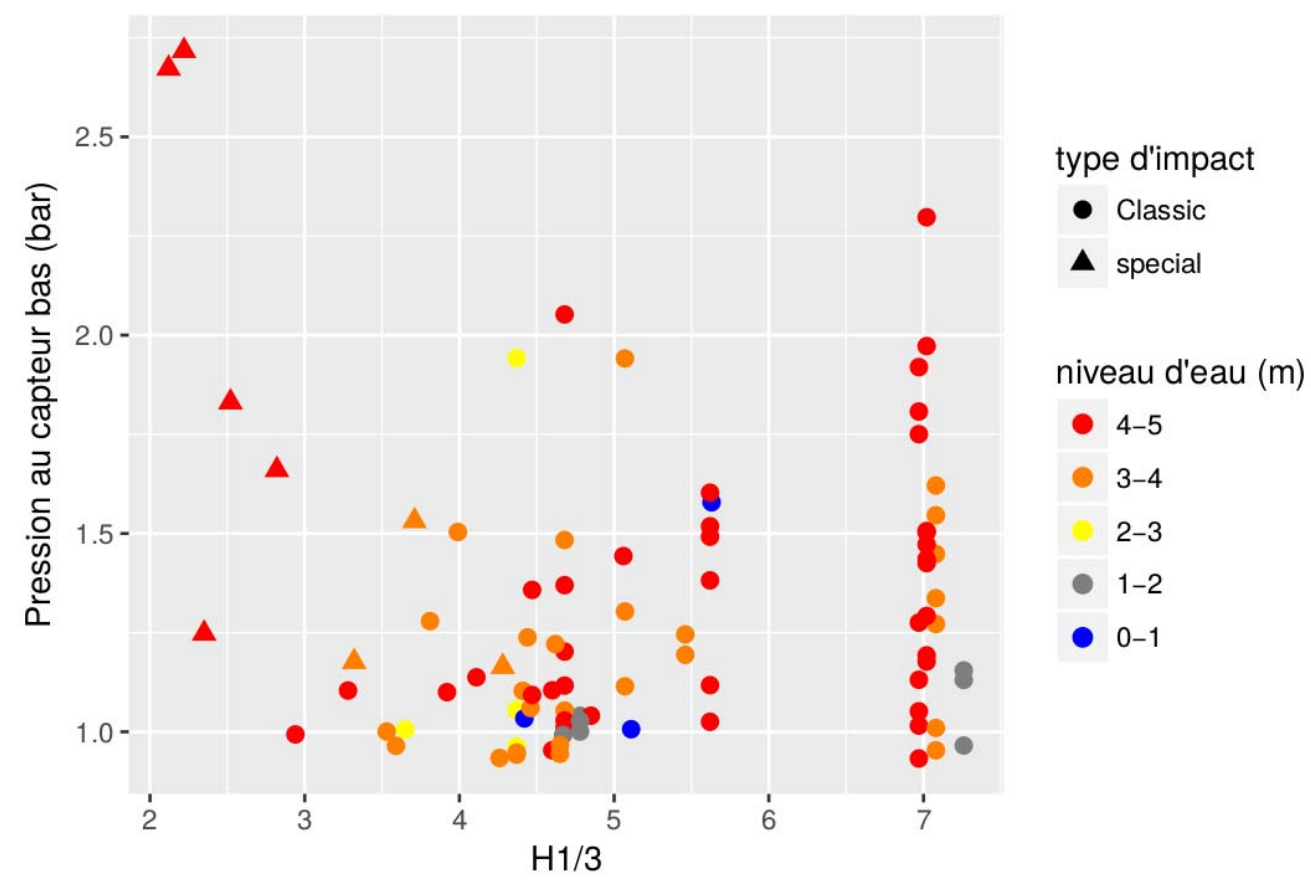

Figure 4. Répartition des impacts en fonction des conditions de houle et du niveau d'eau. Les impacts atypiques sont représentés par des triangles alors que les impacts classiques sont représentés par des ronds. Les couleurs en rouge indiquent des niveaux d'eau plus élevés et bleu plus bas. 


\section{XVèmes Journées Nationales Génie Côtier - Génie Civil \\ La Rochelle, 29 au 31 mai 2018}

Une limite de cette première expérience est l'utilisation de seulement deux capteurs. Il est bien connu (BULLOCK et al., 2001) que les pics de pression élevés n'apparaissent que très localement. Il est donc probable que des valeurs de pression plus élevées puissent être obtenues par un réseau de capteurs plus dense. De plus, un réseau de capteurs couvrant la direction verticale et horizontale peut aussi aider à déterminer le type d'impact.

Les impacts présentant un rapport pic percussif/pallier hydrostatique très fort sont observés dans des conditions de houle relativement peu énergétique et des niveaux d'eau élevés. Une limite de cet indice est qu'il utilise la valeur $\mathrm{H}_{1 / 3}$ comme référence pour la pression hydrostatique. Or la hauteur de la vague qui a générée l'impact peut s'écarter de cette valeur. Ces impacts ressemblent en typologie aux impacts à forte aération décrit dans BULLOCK (2007) avec en particulier des oscillations très marquées après le pic (avec des fréquences dont les valeurs sont assez similaires à celles simulées dans PLUMERAULT et al., (2012)). Il y a parmi ces impacts les deux pics de pression les plus forts du jeu de donnée. Or BULLOCK (2007) prévoit que les pressions les plus fortes soient générées dans des conditions de faible aération, ce qui semble en contradiction avec nos mesures. Néanmoins les mesures réalisées à l'Artha sont pour l'instant limitées à deux capteurs des pressions. Il est donc possible que des impacts faible aération, s'ils existent, aient généré des pressions plus fortes que celles enregistrées mais pas au point des capteurs. A noter que les impacts hautes et faibles aération présentent une distribution spatiale différente (HOFLAND et al. 2011; PLUMERAULT et al. 2012).

\section{Conclusions}

Les résultats préliminaires d'une nouvelle campagne de mesures in-situ de pression d'impact des vagues sur un brise-lame composite situé sur la côte basque française à Saint Jean de Luz (Golfe de Gascogne) ont été présentés dans cet article. Les résultats suivants ont été obtenus: des mesures continues de bonne qualité ont été effectuées de janvier à mars 2016. Les pressions maximales mesurées lors de cette campagne sont plus faibles que dans les précédentes expériences en canal ou par modélisation numériques publiées. Une étude du lien entre les pressions maximales et les paramètres environnementaux montrent le rôle important de la hauteur des vagues et du niveau d'eau. Ce travail doit être poursuivi pour chaque impacts avec en particuliers la reconstitution de la surface libre devant la digue juste avant chaque impact. Une première analyse typologique est proposée. Pour permettre une analyse plus complète, un véritable réseau 2D de 22 capteurs de pression couvrant les directions horizontale et verticale sera installé durant l'hiver 2017-2018.

\section{Références bibliographiques}


ARNOUX F., ABADIE S., BERTIN X., KOJADINOVIK I. (2018). A database to study storm impact statistics along the Basque Coast. International Coastal Symposium (ICS) 2018, Busan, South Korea, Journal of Coastal Research, Special Issue N85.

BIRD P.A.D., CRAWFORD A.R., HEWSON P.J., BULLOCK G.N. (1998). An instrument for field measurement of wave impact pressures and seawater aeration. Coastal Engineering, Vol. 35(1998), pp 103-122. https://doi.org/10.1016/S0378-3839(98)00020-9 BOUGIS J., RIHOUEY D., BERNARD S., VERGNET C., CAYROL C., GARCIA N., JOCOU F., ROUDIL A. (2016). Extrapolation d'essais d'impacts de la houle sur modèles réduits. JNGCGC Toulon 2016, pp 333-342. https://doi.org/10.5150/jngcgc.2016.038 BULLOCK G.N., CRAWFORD A.R., HEWSON P.J., WALKDEN M.J.A., BIRD P.A.D. (2001). The influence of air and scale on wave impact pressures. Coastal Engineering, Vol. 42(4), pp 291-312. https://doi.org/10.1016/S0378-3839(00)00065-X

BULlOCK G.N., OBHRAI C., PEREGRINE D.H., BREDMOSE H. (2007). Violent breaking wave impacts. part 1: Results from large-scale regular wave tests on vertical and sloping walls. Coastal Engineering, Vol. 54(8), pp 602-617. https://doi.org/10.1016/j.coastaleng.2006.12.002

COOKER M.J., PEREGRINE D.H. (1992). Wave impact pressure and its effect upon bodies lying on the sea bed. Coastal Engineering, Vol. 18(3), pp 205-229. https://doi.org/10.1016/0378-3839(92)90020-U

COOKER M.J., PEREGRINE D.H. (1990). A model for breaking wave impact pressures. Coastal Engineering Proceedings, Vol. 1(22), pp 1473-1486. https://doi.org/10.1061/9780872627765.112

DE ROUVILle A., BESSON P., PETRY P. (1938). Etat actuel des études internationales sur les efforts dus aux lames. Annales des Ponts et Chaussées VII.

HOFLAND B., KAMINSKI M., WOLTERS G. (2011). Large scale wave impacts on a vertical wall. Coastal Engineering Proceedings, Vol. 1(32), 15 p.

HULL P., MÜLLER G. (2002). An investigation of breaker heights, shapes and pressures. Ocean Engineering, Vol. 29, pp. 59-79. https://doi.org/10.1016/S0029-8018(00)00075-5 KAMINSKI M.L., BOGAERT H. (2009). Full scale sloshing impact tests. 19th International Off shore and Polar Engineering Conference (Osaka, Japan), International Society of Offshore and Polar Engineers.

LARROQUE B., ARNOULD P., LUTHON F., PONCET P. A., RAHALI A., ABADIE S. (2018). In-situ measurements of wave impact pressure on a composite breakwater: preliminary results. International Coastal Symposium (ICS) 2018, Busan, South Korea, Journal of Coastal Research, Special Issue $n^{\circ} 85$.

OUMERACI, H., KLAMMER P., PARTENSCKY H.W. (1993). Classification of breaking wave loads on vertical structures. Journal of Waterway, Port, Coastal and Ocean Engineering, ASCE, Vol. 119(4), pp 381-397. https://doi.org/10.1061/(ASCE)0733950X(1993)119:4(381) 


\section{XVèmes Journées Nationales Génie Côtier - Génie Civil \\ La Rochelle, 29 au 31 mai 2018}

PEREGRINE D. H. (2003). Water-wave impact on walls. Annual Review of Fluid Mechanics, Vol. 35(1), pp 23-43. https://doi.org/10.1146/annurev.fluid.35.101101.161153

PLUMERAULT L.-R., ASTRUC D., MARON P. (2012). The influence of air on the impact of a plunging breaking wave on a vertical wall using a multifluid model. Coastal Engineering, Vol. 62, pp 62-74. https://doi.org/10.1016/j.coastaleng.2011.12.002

WAGNER H., 1932. The phenomena of impact and planing on water. NACA translation, 1366. 
Thème 3 - Instrumentation, mesures, imagerie et télédétection 